\title{
Optimum Linear Codes with Support Constraints over Small Fields
}

\author{
Hikmet Yildiz, Babak Hassibi \\ California Institute of Technology \\ Email: \{hyildiz, hassibi\}@caltech.edu
}

\begin{abstract}
We consider the problem of designing optimal linear codes (in terms of having the largest minimum distance) subject to a support constraint on the generator matrix. We show that the largest minimum distance can be achieved by a subcode of a Reed-Solomon code of small field size. As a by-product of this result, we settle the GM-MDS conjecture of Dau et. al. in the affirmative.
\end{abstract}

\section{INTRODUCTION}

The problem of designing a linear code with the largest possible minimum distance, subject to support constraints on the generator matrix, has recently found several applications. These include multiple access networks [3], [5] as well as weakly secure data exchange [4], [8]. A simple upper bound on the maximum minimum distance can be obtained from a sequence of Singleton bounds (see eq. (3) below) and can further be achieved by randomly choosing the nonzero elements of the generator matrix from a field of a large enough size.

A natural question to ask is whether the above maximum minimum distance can be achieved with a field of small size, and in particular with a structured, possible algebraic, construction. This question is equivalent to a recently proposed conjecture by Dau et al. [2], which is commonly referred to as the GM-MDS conjecture.

In the past couple of years, progress has been reported on this conjecture. Heidarzadeh et al. [9] have proved it for dimensions $k \leq 5$. Halbawi et al. [5] have proved the statement for $m \leq 3$ if there are $m$ distinct support sets on the rows of the generator matrix. In the authors' previous work [1], the statement has been proved for $m \leq 6$. Halbawi et al. [6], [7] and Song et al. [10], have studied the problem when the generator matrix is sparsest and balanced and established the conjecture in this special case. Yan et al. [8] give some partial results.

In this paper we show that the largest minimum distance can be achieved by a subcode of a Reed-Solomon code of small field size, in fact as low as $2 n-d$, where $n$ is the code length, and $d$ is the maximum minimum distance dictated by the support constraints. As a by-product of this result, we settle the GM-MDS conjecture in the affirmative.

The remainder of the paper is organized as follows. In Section II, we characterize the generator matrices of subcodes of Reed-Solomon codes. Section III defines the problem (of maximizing $d_{\min }$ subject to support constraints) and shows that it can be reduced to the GM-MDS conjecture. Section
IV proposes a more general statement of the problem, that is not directly related to the coding problem, but that more readily lends itself to an induction argument. This is the result we prove which, by fiat, solves the problem of maximizing $d_{\text {min }}$ and the GM-MDS conjecture. The proof is detailed in the Appendix.

\section{A. Notation}

Matrices are shown by bold capital letters and vectors are shown by bold lower case letters. For $n \geq 0$, we denote by $[n]$ the set $\{1,2, \ldots, n\}$ by admitting $[0]=\emptyset$. For $n \geq 1$, we write $\left[\theta_{i}\right]_{i=1}^{m}$ to represent the ordered list of objects $\theta_{1}, \ldots, \theta_{n}$. For a finite nonempty $S \subset \mathbb{Z},\left[\theta_{i}\right]_{i \in S}$ is the ordered list of $\theta_{i}$ 's for $i \in S$ in the ascending order of their indices.

$\mathbb{F}[x]$ represents the polynomial ring over the field $\mathbb{F}$, i.e. the set of polynomials with coefficients in $\mathbb{F} . \mathbb{F}(x)$ represents the field of rational functions in $x$ over the field $\mathbb{F}$, i.e. the set of functions that can be written as a ratio of two polynomials in $\mathbb{F}[x]$ such that the denominator is not the zero polynomial.

$[n, k]_{q}$ and $[n, k, d]_{q}$ represent a linear code over $\mathbb{F}_{q}$ with length $n$, dimension $k$, and minimum distance $d$.

\section{Subcodes of ReEd-Solomon Codes}

An $[n, \ell, n-\ell+1]_{q}$ Reed-Solomon code can be generated by a Vandermonde matrix

$$
\mathbf{V}=\left(\begin{array}{cccc}
1 & 1 & \cdots & 1 \\
\alpha_{1} & \alpha_{2} & \cdots & \alpha_{n} \\
\vdots & \vdots & & \vdots \\
\alpha_{1}^{\ell-1} & \alpha_{2}^{\ell-1} & \cdots & \alpha_{n}^{\ell-1}
\end{array}\right) \in \mathbb{F}_{q}^{\ell \times n}
$$

for distinct $\alpha_{1}, \ldots, \alpha_{n} \in \mathbb{F}_{q}$. Reed-Solomon codes have efficient decoders that can correct up to $\left\lfloor\frac{n-\ell+1}{2}\right\rfloor$ errors.

For $n \geq \ell \geq k,[n, k]_{q}$ subcodes of $[n, \ell]_{q}$ Reed-Solomon codes have generator matrices of the following form:

$$
\mathbf{G}=\mathbf{T} \cdot \mathbf{V}
$$

where $\mathbf{T} \in \mathbb{F}_{q}^{k \times \ell}$ is full rank and $\mathbf{V}$ is given in (1).

The minimum distance $d$ is equal to the minimum weight of $\mathbf{m G}$ over all nonzero row vectors $\mathbf{m} \in \mathbb{F}_{q}^{k}$. Since $\mathbf{V}$ is a Vandermonde matrix, if we treat the entries of $\mathbf{m T}$ as coefficients of a nonzero polynomial $p \in \mathbb{F}_{q}[x]$, then, the entries of $\mathbf{m G}$ will be $p\left(\alpha_{1}\right), \ldots, p\left(\alpha_{n}\right)$. As $\operatorname{deg} p \leq l-1$, the number of nonzero entries in $\mathbf{m G}$ is at least $n-\ell+1$. Therefore, the minimum distance is bounded by $d \geq n-\ell+1$. 
We should mention that every $[n, k]_{q}$ linear code is a subcode of an $[n, n]_{q}$ Reed-Solomon code. However, we are interested in the subcodes of Reed-Solomon codes with the same minimum distance. In other words, we want to design $\ell, \mathbf{T}$, and $\mathbf{V}$ such that $d=n-\ell+1$. Note that in that case, we can use the same decoder of the Reed-Solomon code with the generator matrix $\mathbf{V}$ to correct up to $\left\lfloor\frac{d}{2}\right\rfloor$ errors.

\section{Support Constraint On the Generator Matrix}

Let $S_{1}, S_{2}, \ldots, S_{k} \subset[n]$. For an $[n, k]_{q}$ linear code, suppose that we have the support constraints $\forall i \in[k], \forall j \in S_{i}, \mathbf{G}_{i j}=0$ on the generator matrix $\mathbf{G}$. We are interested in finding a linear code having the largest minimum distance under these constraints.

For any nonempty $\Omega \subset[k]$, the rows of $\mathbf{G}$ indexed in $\Omega$ have zeros in all their entries indexed in $\bigcap_{i \in \Omega} S_{i}$. Consider the submatix of $\mathbf{G}$ consisting of the rows indexed in $\Omega$ and the columns indexed in $[n]-\bigcap_{i \in \Omega} S_{i}$. The minimum distance $d$ of $\mathbf{G}$ is at most the minimum distance of the code generated by this submatrix, which is at most $n-\left|\bigcap_{i \in \Omega} S_{i}\right|-|\Omega|+1$ by the Singleton bound. Hence,

$$
d \leq n+1-\max _{\emptyset \neq \Omega \subset[k]}\left|\bigcap_{i \in \Omega} S_{i}\right|+|\Omega|
$$

Theorem 1 states that we can achieve this bound using the subcodes of Reed-Solomon Codes if $q$ is large enough. In the proof of Theorem 1, we will show that it is enough to prove a special case, Theorem 2, where $\ell=k$ and the optimum code is an MDS code.

Theorem 1. Let $S_{1}, S_{2}, \ldots, S_{k} \subset[n]$,

$$
\ell \triangleq \max _{\emptyset \neq \Omega \subset[k]}\left|\bigcap_{i \in \Omega} S_{i}\right|+|\Omega|
$$

and $q \geq n+\ell-1$ be a field size. Then, there exists an $[n, k, d]_{q}$ subcode of a Reed-Solomon code that achieves $d=n-\ell+1$ such that the generator matrix $\mathbf{G}$ has zeros at $(i, j)$ for $j \in S_{i}, i \in[k]$.

Proof. For $\Omega=[k]$, we have $\ell \geq k$. Let $S_{k+1}, \ldots, S_{\ell}=\emptyset$. By Theorem 2, there exists an $[n, \ell]_{q}$ Reed-Solomon code that has a generator matrix $\mathbf{G}^{\prime}$ such that $\mathbf{G}_{i j}^{\prime}=0$ for $j \in S_{i}, i \in[\ell]$. The subcode with the generator matrix $\mathbf{G}$ consisting of the first $k$ rows of $\mathbf{G}^{\prime}$ satisfies the desired constraints.

\section{A. Existence of MDS codes}

As a special case, we will describe necessary and sufficient conditions on the support constraints for the existence of a Reed-Solomon code whose generator matrix satisfies these support constraints. Theorem 2 has been known as the GMMDS conjecture, which is proposed by Dau et al. in [2], where they showed that it is equivalent to Corollary 1 given in Section IV.

Theorem 2. Let $S_{1}, S_{2}, \ldots, S_{k} \subset[n]$ and $q \geq n+k-1$ be a field size. For any nonempty $\Omega \subset[k]$,

$$
\left|\bigcap_{i \in \Omega} S_{i}\right| \leq k-|\Omega|
$$

if and only if there exists an $[n, k]_{q}$ Reed-Solomon code with a generator matrix $\mathbf{G}$ such that $\mathbf{G}_{i j}=0$ for $j \in S_{i}, i \in[k]$.

\section{MAIN THEOREM}

For $n \geq 0$, let $\mathbb{K}_{n}=\mathbb{Q}\left(\alpha_{1}, \ldots, \alpha_{n}\right)$ be the field of rational functions in $\alpha_{1}, \ldots, \alpha_{n}$ over the set of rational numbers. We will admit that $\mathbb{K}_{0}=\mathbb{Q}$. For $k \geq m \geq 1$ and $n \geq 0$, define

$$
\begin{gathered}
\mathcal{S}_{k, m, n}=\left\{\left[\left(S_{i}, r_{i}\right)\right]_{i=1}^{m} \mid \forall i \in[m] S_{i} \subset[n], r_{i} \in \mathbb{Z}^{+},\right. \\
\left.\left|S_{i}\right|+r_{i} \leq k, \quad \sum_{i=1}^{m} r_{i}=k\right\}
\end{gathered}
$$

Define the matrix $\mathbf{M} \in \mathbb{K}_{n}^{k \times k}$ in terms of the parameters $\left[\left(S_{i}, r_{i}\right)\right]_{i=1}^{m} \in \mathcal{S}_{k, m, n}$ as follows (We will often write $\mathbf{M}\left[\left(S_{i}, r_{i}\right)\right]_{i=1}^{m}$ to indicate its parameters):

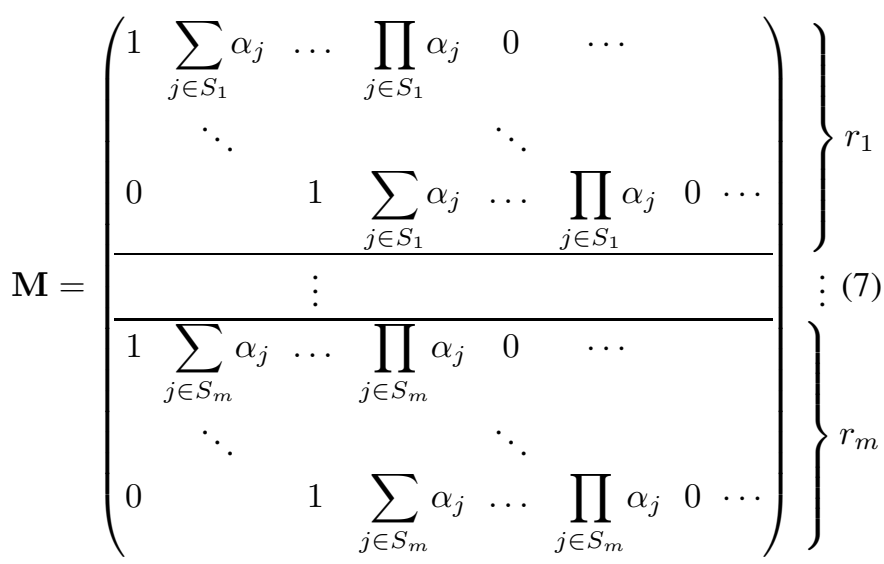

The rows are partitioned into $m$ blocks and for $i \in[m]$, the $i$ th block is an $r_{i} \times k$ upper triangular Toeplitz matrix, whose first row consists of the coefficients of the polynomial $x^{k-\left|S_{i}\right|-1} \prod_{j \in S_{i}}\left(x+\alpha_{j}\right)$ in descending order with respect to the degree. This matrix can be also thought of as a generalized Sylvester matrix that is constructed by $m$ polynomials. The condition $\left|S_{i}\right|+r_{i} \leq k$ ensures that the rows are not shifted too much that we lose a nonzero entry in the last row of the $i$ th block. Also, notice that the bottom-right entry of the $i$ th block is nonzero if we have the equality $\left|S_{i}\right|+r_{i}=k$, and zero otherwise. So, we need at least one equality if we want $\mathrm{M}$ to be nonsingular.

In Proposition 1, we give an equivalent way of writing $\operatorname{det} \mathbf{M}=0$ in terms of the polynomials that we use when constructing $\mathbf{M}$.

Proposition 1. Let $\left[\left(S_{i}, r_{i}\right)\right]_{i=1}^{m} \in \mathcal{S}_{k, m, n}$. For $i \in[m]$, define

$$
p_{i}=x^{k-\left|S_{i}\right|-r_{i}} \prod_{j \in S_{i}}\left(x+\alpha_{j}\right)
$$

Then, $\operatorname{det} \mathbf{M}\left[\left(S_{i}, r_{i}\right)\right]_{i=1}^{m}=0$ if and only if there exist $q_{1}, \ldots, q_{m} \in \mathbb{K}_{n}[x]$, not all zero, such that $\operatorname{deg} q_{i} \leq r_{i}-1$ for $i \in[m]$ and $\sum_{i=1}^{m} p_{i} q_{i}=0$.

Proof. For each $q_{i}$, construct a row vector of size $r_{i}$ consisting of the coefficients of $x^{r_{i}-1}, \ldots, x, 1$ in $q_{i}$ and merge them into one row vector $\mathbf{y} \in \mathbb{K}_{n}^{1 \times k}$. Then, $\sum_{i=1}^{m} p_{i} q_{i}=0$ iff $\mathbf{y} \cdot \mathbf{M}=0$. The statement follows from $\operatorname{det} \mathbf{M}=0$ iff there exists nonzero $\mathbf{y} \in \mathbb{K}_{n}^{1 \times k}$ such that $\mathbf{y} \cdot \mathbf{M}=0$. 
Theorem 3 gives necessary and sufficient conditions on the parameters $\left[\left(S_{i}, r_{i}\right)\right]_{i=1}^{m}$ for $\operatorname{det} \mathbf{M}$ to be nonzero.

Theorem 3. Let $k \geq m \geq 1, n \geq 0,\left[\left(S_{i}, r_{i}\right)\right]_{i=1}^{m} \in \mathcal{S}_{k, m, n}$. Then, $\operatorname{det} \mathbf{M}\left[\left(S_{i}, r_{i}\right)\right]_{i=1}^{m} \neq 0$ if and only if for any nonempty $\Omega \subset[m]$,

$$
\left|\bigcap_{i \in \Omega} S_{i}\right|+\sum_{i \in \Omega} r_{i} \leq \max _{i \in \Omega}\left|S_{i}\right|+r_{i}
$$

\section{Proof. See Appendix A.}

In Theorem 3, if we let $k=m, r_{i}=1$, and $\left|S_{i}\right|=k-1$, we will get Corollary 1.

Corollary 1. Let $S_{1}, S_{2}, \ldots, S_{k} \subset[n]$ such that $\left|S_{i}\right|=k-1$. Then, the determinant of

$$
\mathbf{M}\left[\left(S_{i}, 1\right)\right]_{i=1}^{k}=\left(\begin{array}{cccc}
1 & \sum_{j \in S_{1}} \alpha_{j} & \cdots & \prod j \in S_{1} \alpha_{j} \\
1 & \sum_{j \in S_{2}} \alpha_{j} & \cdots & \prod j \in S_{2} \alpha_{j} \\
\vdots & \vdots & & \vdots \\
1 & \sum_{j \in S_{m}} \alpha_{j} & \cdots & \prod j \in S_{m} \alpha_{j}
\end{array}\right)
$$

is nonzero if and only if for any nonempty $\Omega \subset[k]$,

$$
\left|\bigcap_{i \in \Omega} S_{i}\right| \leq k-|\Omega|
$$

\section{APPENDIX A}

\section{PROOF OF THEOREM 3}

Suppose that for some nonempty $\Omega \subset[m]$, the condition (9) is not true. Let $S_{0}=\bigcap_{i \in \Omega} S_{i}, r_{0}=\sum_{i \in \Omega} r_{i}$, $k^{\prime}=\max _{i \in \Omega}\left|S_{i}\right|+r_{i}$. Then, $\left|S_{0}\right|+r_{0}>k^{\prime}$. Consider the $r_{0}$ rows of $\mathbf{M}$ in the blocks indexed in $\Omega$. They all have zeros in their last $k-k^{\prime}$ entries. Let $\mathbf{M}_{0} \in \mathbb{K}_{n}^{r_{0} \times k^{\prime}}$ be the submatrix consisting of these rows without including the last $k-k^{\prime}$ columns. We will prove that rank $\mathbf{M}_{0}<r_{0}$, which implies $\operatorname{det} \mathbf{M}=0$. Let $\mathbf{W}=\left(\left(-\alpha_{j}\right)^{1-i}\right)_{i \in\left[k^{\prime}\right], j \in S_{0}}$ be $k^{\prime} \times\left|S_{0}\right|$ Vandermonde matrix. Then, $\mathbf{M}_{0} \cdot \mathbf{W}=0$ because the polynomials with the coefficients in the rows of $\mathbf{M}_{0}$ vanish at $-\alpha_{j}$ for $j \in S_{0}$. Hence,

$$
\operatorname{rank} \mathbf{M}_{0} \leq k^{\prime}-\operatorname{rank} \mathbf{W} \leq k^{\prime}-\min \left\{k^{\prime},\left|S_{0}\right|\right\}<r_{0}
$$

which proves the first direction.

For the other direction, we will apply induction on the parameters $(k, m, n)$ considered in the lexicographical order. For $m=1, \mathcal{S}_{k, 1, n}=\{[(\emptyset, k)]\}$ and $\operatorname{det} \mathbf{M}[(\emptyset, k)]=\operatorname{det} \mathbf{I}_{k}=1$. For $n=0$, all of $S_{i}$ 's are empty; hence, for $\Omega=[m]$, (9) yields $m=1$, for which, we already showed $\operatorname{det} \mathbf{M}=1$. For $k \geq m \geq 2$ and $n \geq 1$, assume that the statement is true for parameters $\left(k^{\prime}, m^{\prime}, n^{\prime}\right)$ that are smaller than $(k, m, n)$ with respect to lexicographical order. Take any $\left[\left(S_{i}, r_{i}\right)\right]_{i=1}^{m} \in \mathcal{S}_{k, m, n}$ that satisfies the condition (9). We will prove that $\operatorname{det} \mathbf{M}\left[\left(S_{i}, r_{i}\right)\right]_{i=1}^{m} \neq 0$ under three cases:

1) There exists $\Omega_{1} \subset[m]$ such that $2 \leq\left|\Omega_{1}\right| \leq m-1$ and

$$
\left|\bigcap_{i \in \Omega_{1}} S_{i}\right|+\sum_{i \in \Omega_{1}} r_{i}=\max _{i \in \Omega_{1}}\left|S_{i}\right|+r_{i}
$$

2) There exists a unique $i \in[m]$ such that $\left|S_{i}\right|+r_{i}=k$.

3) Else (i.e. 1 and 2 are false).

\section{Case 1}

Let $\Omega_{2}=\{0\} \cup[m]-\Omega_{1}$. Note that $2 \leq\left|\Omega_{1}\right|,\left|\Omega_{2}\right| \leq m-1$. Define

$$
S_{0}=\bigcap_{i \in \Omega_{1}} S_{i}, \quad r_{0}=\sum_{i \in \Omega_{1}} r_{i}
$$

Then, (13) becomes

$$
\left|S_{0}\right|+r_{0}=\max _{i \in \Omega_{1}}\left|S_{i}\right|+r_{i}
$$

Define $S_{i}^{\prime}=S_{i}-S_{0}$ for $i \in \Omega_{1}$. Then,

$$
\left[\left(S_{i}^{\prime}, r_{i}\right)\right]_{i \in \Omega_{1}} \in \mathcal{S}_{r_{0},\left|\Omega_{1}\right|, n}, \quad\left[\left(S_{i}, r_{i}\right)\right]_{i \in \Omega_{2}} \in \mathcal{S}_{k,\left|\Omega_{2}\right|, n}
$$

The first one is true because $r_{0}=\sum_{i \in \Omega_{1}} r_{i}$ and for any $i \in \Omega_{1}$, by (15),

$$
\left|S_{i}^{\prime}\right|+r_{i}=\left|S_{i}\right|+r_{i}-\left|S_{0}\right| \leq r_{0}
$$

The second one is true because

$$
k=\sum_{i=1}^{m} r_{i}=\sum_{i \in \Omega_{1}} r_{i}+\sum_{i \in[m]-\Omega_{1}} r_{i}=\sum_{i \in \Omega_{2}} r_{i},
$$

$\left|S_{i}\right|+r_{i} \leq k$ for $i \in[m]-\Omega_{1}$ and $\left|S_{0}\right|+r_{0} \leq k$ due to (15).

By the induction hypothesis, the statement is true for $\left[\left(S_{i}^{\prime}, r_{i}\right)\right]_{i \in \Omega_{1}}$ and $\left[\left(S_{i}, r_{i}\right)\right]_{i \in \Omega_{2}}$. We will show that both satisfy the condition (9):

1) For any nonempty $\Omega \subset \Omega_{1}$,

$$
\begin{aligned}
\left|\bigcap_{i \in \Omega} S_{i}^{\prime}\right|+\sum_{i \in \Omega} r_{i} & =\left|\bigcap_{i \in \Omega} S_{i}\right|-\left|S_{0}\right|+\sum_{i \in \Omega} r_{i} \\
& \leq \max _{i \in \Omega}\left|S_{i}\right|+r_{i}-\left|S_{0}\right| \\
& =\max _{i \in \Omega}\left|S_{i}^{\prime}\right|+r_{i}
\end{aligned}
$$

2) For any nonempty $\Omega \subset \Omega_{2}$, if $0 \notin \Omega_{2}$, then $\Omega \subset[m]$ and (9) holds trivially. Assume $\Omega=\{0\} \cup \Omega^{\prime}$ for some $\Omega^{\prime} \subset[m]-\Omega_{1}$. Then,

$$
\begin{aligned}
\left|\bigcap_{i \in \Omega} S_{i}\right|+\sum_{i \in \Omega} r_{i} & =\left|\bigcap_{i \in \Omega_{1} \cup \Omega^{\prime}} S_{i}\right|+\sum_{i \in \Omega_{1} \cup \Omega^{\prime}} r_{i} \\
& \leq \max _{i \in \Omega_{1} \cup \Omega^{\prime}}\left|S_{i}\right|+r_{i} \\
& =\max _{i \in \Omega}\left|S_{i}\right|+r_{i}
\end{aligned}
$$

Hence, we have that

$$
\operatorname{det} \mathbf{M}\left[\left(S_{i}^{\prime}, r_{i}\right)\right]_{i \in \Omega_{1}} \neq 0, \quad \operatorname{det} \mathbf{M}\left[\left(S_{i}, r_{i}\right)\right]_{i \in \Omega_{2}} \neq 0
$$

Now, we will use Proposition 1. Define for $i \in\{0\} \cup[m]$,

$$
p_{i}=x^{k-\left|S_{i}\right|-r_{i}} \prod_{j \in S_{i}}\left(x+\alpha_{j}\right)
$$

and for $i \in \Omega_{1}$,

$$
p_{i}^{\prime}=x^{r_{0}-\left|S_{i}^{\prime}\right|-r_{i}} \prod_{j \in S_{i}^{\prime}}\left(x+\alpha_{j}\right)
$$

Note that for $i \in \Omega_{1}, p_{i}=p_{i}^{\prime} p_{0}$. 
Consider any $q_{1}, \ldots, q_{m} \subset \mathbb{K}_{n}[x]$ such that $\operatorname{deg} q_{i} \leq r_{i}-1$ for $i \in[m]$ and $\sum_{i=1}^{m} p_{i} q_{i}=0$. We need to prove that $q_{i}=0$ for all $i \in[m]$. Define

$$
q_{0}=\sum_{i \in \Omega_{1}} p_{i}^{\prime} q_{i}
$$

Note that $\operatorname{deg} q_{0} \leq r_{0}-1$ :

$$
\begin{aligned}
\operatorname{deg} q_{0} & \leq \max _{i \in \Omega_{1}}\left(\operatorname{deg} p_{i}^{\prime}+\operatorname{deg} q_{i}\right) \\
& \leq \max _{i \in \Omega_{1}}\left(\left(r_{0}-r_{i}\right)+\left(r_{i}-1\right)\right) \\
& =r_{0}-1
\end{aligned}
$$

Also, we can write that

$$
0=\sum_{i=1}^{m} p_{i} q_{i}=p_{0} \sum_{i \in \Omega_{1}} p_{i}^{\prime} q_{i}+\sum_{i \in[m]-\Omega_{1}} p_{i} q_{i}=\sum_{i \in \Omega_{2}} p_{i} q_{i}
$$

Then, by Proposition 1, we get $q_{i}=0$ for all $i \in \Omega_{2}$. Then, $q_{0}=\sum_{i \in \Omega_{1}} p_{i}^{\prime} q_{i}=0$. Then, by Proposition $1, q_{i}=0$ for all $i \in \Omega_{1}$. Hence, $q_{i}=0$ for all $i \in[m]$. By Proposition 1, $\operatorname{det} \mathbf{M}\left[\left(S_{i}, r_{i}\right)\right]_{i=1}^{m} \neq 0$.

\section{Case 2}

W.l.o.g., let $m$ be the maximizer. Then, for $i \in[m-1]$,

$$
k=\left|S_{m}\right|+r_{m}>\left|S_{i}\right|+r_{i}
$$

Then, the last column of $\mathbf{M}\left[\left(S_{i}, r_{i}\right)\right]_{i=1}^{m}$ is all zero except the last entry, which is $\prod_{j \in S_{m}} \alpha_{j}$.

Hence, we have

$$
\operatorname{det} \mathbf{M}\left[\left(S_{i}, r_{i}\right)\right]_{i=1}^{m}=\operatorname{det} \mathbf{M}\left[\left(S_{i}, r_{i}^{\prime}\right)\right]_{i=1}^{m} \cdot \prod_{j \in S_{m}} \alpha_{j}
$$

where $r_{m}^{\prime}=r_{m}-1$ and $r_{i}^{\prime}=r_{i}$ for $i \in[m-1]$ assuming that $r_{m} \geq 2$. (If $r_{m}=1$, the first multiplier would be $\operatorname{det} \mathbf{M}\left[\left(S_{i}, r_{i}\right)\right]_{i=1}^{m-1}$, which is nonzero by the induction hypothesis.)

Note that $\left[\left(S_{i}, r_{i}^{\prime}\right)\right]_{i=1}^{m} \in \mathcal{S}_{k-1, m, n}$ since $\sum_{i=1}^{m} r_{i}^{\prime}=k-1$ and $\left|S_{i}\right|+r_{i}^{\prime} \leq k-1$ for any $i \in[m]$ due to the unique maximizer assumption.

By the induction hypothesis, the statement is true for $\left[\left(S_{i}, r_{i}^{\prime}\right)\right]_{i=1}^{m}$. If we prove that it satisfies the condition (9), then $\operatorname{det} \mathbf{M}\left[\left(S_{i}, r_{i}\right)\right]_{i=1}^{m} \neq 0$.

For any nonempty $\Omega \subset[m]$, if $m \notin \Omega$, then (9) holds trivially. Assume $m \in \Omega$.

$$
\begin{aligned}
\left|\bigcap_{i \in \Omega} S_{i}\right|+\sum_{i \in \Omega} r_{i}^{\prime} & =\left|\bigcap_{i \in \Omega} S_{i}\right|-1+\sum_{i \in \Omega} r_{i} \\
& \leq \max _{i \in \Omega}\left|S_{i}\right|+r_{i}-1 \\
& =k-1 \\
& =\max _{i \in \Omega}\left|S_{i}\right|+r_{i}^{\prime}
\end{aligned}
$$

\section{Case 3}

For any nonempty $\Omega \subset[m]$ such that $|\Omega| \neq 1, m$, we have

$$
\left|\bigcap_{i \in \Omega} S_{i}\right|+\sum_{i \in \Omega} r_{i} \leq \max _{i \in \Omega}\left|S_{i}\right|+r_{i}-1
$$

Also, there exist at least two maximizers of $\left|S_{i}\right|+r_{i}$. W.l.o.g., assume that

$$
k=\left|S_{m}\right|+r_{m}=\left|S_{m-1}\right|+r_{m-1}
$$

If $S_{m}=S_{m-1}$, we get a contradiction in (9):

$$
r_{m}+r_{m-1} \leq \max \left\{r_{m}, r_{m-1}\right\}
$$

Then, either $S_{m-1} \neq[n]$ or $S_{m} \neq[n]$. W.l.o.g., we can assume that $n \notin S_{m}$. Substitute $\alpha_{n}=0$ :

$$
\left.\operatorname{det} \mathbf{M}\left[\left(S_{i}, r_{i}\right)\right]_{i=1}^{m}\right|_{\alpha_{n}=0}=\operatorname{det} \mathbf{M}\left[\left(S_{i}^{\prime}, r_{i}\right)\right]_{i=1}^{m}
$$

where $S_{i}^{\prime}=S_{i}-\{n\}$.

Note that $\left[\left(S_{i}^{\prime}, r_{i}\right)\right]_{i=1}^{m} \in \mathcal{S}_{k, m, n-1}$ since $S_{i}^{\prime} \subset[n-1]$ and $\left|S_{i}^{\prime}\right|+r_{i} \leq\left|S_{i}\right|+r_{i} \leq k$ for $i \in[m]$.

By the induction hypothesis, the statement is true for $\left[\left(S_{i}^{\prime}, r_{i}\right)\right]_{i=1}^{m}$. If we prove that it satisfies the condition (9), then $\operatorname{det} \mathbf{M}\left[\left(S_{i}, r_{i}\right)\right]_{i=1}^{m} \neq 0$.

For $|\Omega|=1$, (9) holds trivially. For $|\Omega| \neq 1, m$, we have

$$
\begin{aligned}
\left|\bigcap_{i \in \Omega} S_{i}^{\prime}\right|+\sum_{i \in \Omega} r_{i} & \leq\left|\bigcap_{i \in \Omega} S_{i}\right|+\sum_{i \in \Omega} r_{i} \\
& \leq \max _{i \in \Omega}\left|S_{i}\right|+r_{i}-1 \\
& \leq \max _{i \in \Omega}\left|S_{i}^{\prime}\right|+r_{i}
\end{aligned}
$$

For $\Omega=[m]$, it is enough to show that $k=\max _{i \in[m]}\left|S_{i}^{\prime}\right|+r_{i}$, which is true because

$$
\left|S_{m}^{\prime}\right|+r_{m}=\left|S_{m}\right|+r_{m}=k
$$

\section{REFERENCES}

[1] H. Yildiz and B. Hassibi, "Further progress on the gm-mds conjecture for reed-solomon codes," arXiv preprint arXiv:1801.07865, 2018.

[2] S. H. Dau, W. Song, and C. Yuen, "On the existence of mds codes over small fields with constrained generator matrices," in International Symposium on Information Theory (ISIT). IEEE, 2014, pp. 1787-1791.

[3] S. H. Dau, W. Song, and C. Yuen, "On simple multiple access networks," IEEE Journal on Selected Areas in Communications. vol. 33, no. 2, pp. 236-249, 2015

[4] M. Yan and A. Sprintson, "Algorithms for weakly secure data exchange," in International Symposium on Network Coding (NetCod). IEEE, 2013, pp. $1-6$.

[5] W. Halbawi, T. Ho, H. Yao, and I. Duursma, "Distributed reed-solomon codes for simple multiple access networks," in International Symposium on Information Theory (ISIT). IEEE, 2014, pp. 651-655.

[6] W. Halbawi, Z. Liu, and B. Hassibi, "Balanced reed-solomon codes," in International Symposium on Information Theory (ISIT). IEEE, 2016, pp. 935-939.

[7] W. Halbawi, Z. Liu, and B. Hassibi, "Balanced Reed-Solomon codes for all parameters," in Information Theory Workshop (ITW). IEEE, 2016, pp. 409-413.

[8] M. Yan, A. Sprintson, and I. Zelenko, "Weakly secure data exchange with generalized reed solomon codes," in International Symposium on Information Theory (ISIT). IEEE, 2014, pp. 1366-1370.

[9] A. Heidarzadeh and A. Sprintson, "An algebraic-combinatorial proof technique for the gm-mds conjecture," in International Symposium on Information Theory (ISIT). IEEE, 2017, pp. 11-15.

[10] W. Song and K. Cai, "Generalized reed-solomon codes with sparsest and balanced generator matrices," arXiv preprint arXiv:1801.02315, 2018. 